\title{
Comment exploiter les 'corpus-surprise' ?
}

\author{
Chantal Rittaud-Hutinet (Paris)
}

\begin{abstract}
To what extent non-recorded oral corpora may constitute objects of analysis of pragmatic meaning?

These corpora are heard by chance: on the radio, on television, in the street, a shop, a means of transport or generally in any conversational interaction in which the linguist participates, but had not previously planned to record for his research. The problem of the use of these corpora in linguistics is all the more crucial since the aim, in phonopragmatics, is to discover the functions and significations of their phonic part. I shall attempt to answer the following questions:

- The accuracy of the transcription with respect to the original. To what extent can we ignore our own phonological code, our regional variants, mastered/partly known styles ofspeech?

- The reliability of the oral reproduction carried out by the linguist - for example, during a talk at a conference. What is his capacity for deferred mimicry?

- The relation between a significant discrepancy and the elocutionary habits of the speaker.

- The relation between the comprehension of the external auditors and the effect produced on the 'real' person addressed.
\end{abstract}

Considering that transparency is (sometimes? often?) an illusion, I shall also examine what precautions should be taken so that these corpora offer guarantees as to the veracity.

\section{$1 \quad$ Introduction}

On oublie souvent que l'oralité non préparée exige de son énonciateur de réaliser simultanément de nombreuses tâches - idéation, mise en forme lexicale, grammaticale, phonétique, prise en compte du lieu, de l'interlocuteur, etc. -, car leur complexité ne devient visible que lorsqu'il se produit par exemple une erreur ou un conflit de programmation ou encore qu'un trou de mémoire bloque le flux discursif.

De même, comprendre ce que dit le locuteur ne va pas de soi, c'est une re-création de signification: établissement des cohérences lexicale, morpho-syntaxique, phonétique, textuelle, prise en compte des éléments de la situation, des relations et codes sociétaux, etc. C'est pourquoi se pose la question du traitement des extraits non-enregistrés et de la possibilité de leur exploitation dans les recherches sur les échanges improprement appelés "spontanés". Ces exemples, je les nomme les corpus-surprise. J'entends par là les fragments de conversation entendus par hasard et notés à la volée (comme le faisaient les dialectologues jusqu'au milieu du $\mathrm{XX}^{\mathrm{e}}$ siècle), que ce soit à la radio ou à la télévision, dans la rue, un magasin, un moyen de transport, et en général dans toute interaction conversationnelle que le linguiste n'avait pas programmé d'enregistrer pour sa recherche. 
Le problème de l'utilisation de ces corpus n'est pas moins crucial en sémantique qu'en phonopragmatique $^{1}$, où il s'agit de décortiquer les fonctions et significations de la partie sonore des verbalisations et de décrire les indices acoustiques, segmentaux comme suprasegmentaux, qui leur sont spécifiques (cf. Rittaud-Hutinet, entre autres 1995).

\section{$2 \quad$ Les difficultés}

Je tenterai de répondre aux principales questions qui se posent quant aux items non enregistrés en proposant quelques-unes des procédures que l'on utilise également pour le décryptage perceptuel des items enregistrés, de sorte qu'ils puissent être validés comme objets d'analyse.

\subsection{Le transcodage oral $\rightarrow$ écrit}

Lors du passage à l'écrit, de même que pour la couche lexicale - où les troncations, ruptures, hésitations, reprises et autres 'ratures' doivent toutes sans exception être notées scrupuleusement et avec exactitude -, le premier problème pour la partie sonore du message est celui de sa conformité avec l'actualisation originale.

Avec un corpus enregistré, on a aujourd'hui recours à l'analyse informatique du signal, parfois conjointement avec la perception du chercheur, parfois de façon exclusive ${ }^{2}$, ce qui ne va pas non plus sans difficulté (cf. par exemple Post/Delais-Roussarie/Simon 2006). Mais comment faire par exemple en (1), avec ce spot publicitaire télévisé, donc surpris in situ, donc - pour ce qui est du texte - en ne disposant que de l'audition?

(1) Nouvelle Opel Agila. [...] D'abord ha ha ha! ensuite aha! ${ }^{3}$

= "on ne me la fait pas, à moi! on ne me fera pas croire que ce coffre a une telle capacité!"

= "chapeau! la capacité de ce coffre est bien aussi grande que vous le disiez (en fait: montriez)! très bien, je reconnais et j'admire!"

Même dans ces cas-là - et peut-être d'ailleurs surtout dans ces cas-là -, l'utilisateur-linguiste s'attendra à ce que la transcription soit à la fois objective, pertinente, précise et rigoureuse, c'est-à-dire exacte et détaillée, donc irréprochable et par conséquent fiable. Cela signifie qu'il doit pouvoir faire confiance:

- à la transcription phonétique - en (1) les sons mis en caractères gras -,

- et à la traduction des indices supra-segmentaux - en (1'), les hauteurs tonales.

Ces deux sortes d'information sont restituées en (1'):

\footnotetext{
1 Bases théoriques de cette recherche: certains faits sonores 'font sens', les signes vocaux, unités discursives et dialogales, qui fonctionnent comme des auto-segments; leur emploi relève d'un choix de l'énonciateur, en fonction de ses intentions, buts et stratégies; ils ont des synonymes.

Objectifs: établir la régularité de la relation signifiant vocal/signifié pragmatique; dégager et décrire les traits acoustiques que contiennent ces faits sonores signifiants; étudier leur extension fonctionnelle et situer leurs limites ou zones marginales; cerner leurs lieux d'apparition, leur degré d'autonomie par rapport aux contraintes des composantes phonologique, combinatoire, intonosyntaxique, les rapports qu'ils entretiennent avec le sens du support lexical; déterminer les plans dans lesquels s'appliquent leur signifié.

L'analyse des données tient compte de la pertinence que manifeste le signe vocal par rapport au contexte précédent et/ou à la suite de l'échange langagier, de ses causes et de ses effets, par rapport à l'interlocuteur comme au discours de l'énonciateur.

2 Par exemple : Praat (analyse de la voix), Analor (périodes intonatives), WinPitch (proéminences accentuelles), EasyAlign (alignement automatique : mots graphiques, syllabes, phonèmes, à partir d'une transcription orthographique), Unitex (assistance à l'analyse quantitative et/ou qualitative).

3 Sur M6, 10 septembre 2006.

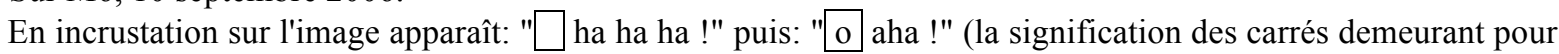
moi un mystère).
} 
$\left(1^{\prime}\right)$

TV nou'velle Opel Agi'la / d'abord

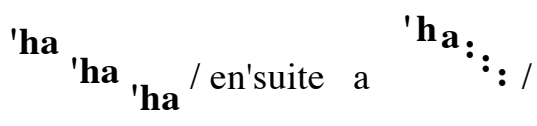

[nu'vel opel azi'la / daboR

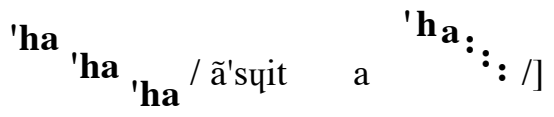

$$
\begin{aligned}
& \begin{array}{l|l|lllllll}
1 \mid & 2 & \mathbf{3} & \mathbf{2} & \mathbf{1} & 2 & 2,5 & 2 & \mathbf{4 , 5} \\
\text { à } 2
\end{array} \\
& \text { B } \mid \begin{array}{llllllll}
\mathbf{M}^{-} & \mathbf{M}^{+} & \mathbf{M}^{-} \mathbf{B} & \mathbf{M}^{-} & \mathrm{M} & \mathbf{M}^{-} & \mathbf{H} \text { à } & \mathbf{M}^{4}
\end{array}
\end{aligned}
$$

On y voit que:

- la transcription est faite soit en orthographe traditionnelle complétée de signes diacritiques pour les accents et pour les pauses, soit en alphabet phonétique intégral;

- les hauteurs tonales peuvent être figurées de 3 façons différentes: en chiffres ${ }^{5}$, en lettres ${ }^{6}$, ou par une schématisation utilisant plusieurs lignes d'écriture par ligne pour représenter visuellement les intonations, de façon approchée.

Dès lors chacun attend de ce travail de notation qu'il soit le miroir de la réalité orale. Mais s'il est fait de façon trop automatique, c'est-à-dire si le linguiste ne fait pas/n'arrive pas à faire/ oublie de faire abstraction de sa grille phonologique, de ses variantes régionales, des styles de parole qu'il maîtrise ou qu'il connaît en partie, de ses habitudes langagières, de son sens de la norme, on ne dispose plus que d'une sorte de schéma d'énoncé fort peu utilisable. Par exemple ce qu'on lit en (2)

(2) je ne sais pas s'ils ont déjà semé le blé

suscite toutes sortes d'interrogations, qui se ramènent à la question suivante: comment l'émetteur a-t-il effectivement réalisé cette réplique? La solution prosodique fournie par (2') apparaît comme une version tout à fait canonique. C'est en effet celle à laquelle on aboutit si la force de la norme morpho-syntaxique intériorisée surpasse l'écoute objective:

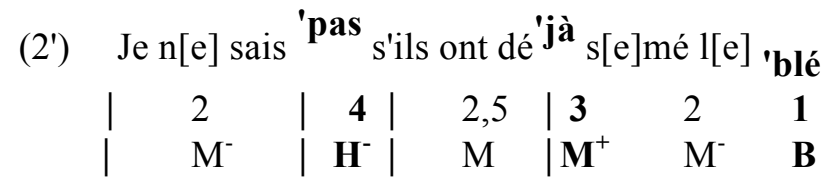

Avec (2'), on comprend que l'énonciateur pose une question indirecte. Mais l'intervenant réel avait, lui, dit tout autre chose, que nous montre (2"),

(2") T- $[\ldots]$ je $n[\mathrm{e}]$ "sais 'pas s'ils ont déjà s[e]mé $1[\mathrm{e}]$ 'blé $[\ldots]^{7}$

\begin{tabular}{lll|l|l}
2 & $\mathbf{4}$ & $\mathbf{1}$ & $\mathbf{1}$ faiblement descendant \\
$\mathbf{M}^{-}$ & $\mathbf{H}^{-}$ & $\mathbf{B}$ & B faiblement descendant
\end{tabular}

où "s'ils ont déjà semé le blé" est une proposition incidente, comparable à une dislocation à droite (réitération d'une information) mais dont le deuxième terme serait le contexte situationnel, car elle double le fait que les deux voyageurs regardaient un champ par la fenêtre. En (2"), c'est "je ne sais pas" qui contient l'ensemble de la réponse de T à son interlocuteur.

À l'évidence, c'est la différence de transcription qui est responsable de la différence entre les messages délivrés respectivement par (2') et par (2").

\footnotetext{
4 Sans traits verticaux, chaque nombre/lettre = une seule syllabe.

5 Notation d'après Morel/Danon-Boileau (1998), légèrement modifiée (voir ci-dessous, note 6).

6 Supra-haut (= hors de la gamme habituelle du locuteur): $\mathrm{SH}, 6$; très haut: $\mathrm{H}^{+}, 5$; haut: $\mathrm{H}, 4,5$; infra-haut: $\mathrm{H}^{-}$, 4; moyen-haut: $\mathrm{M}^{+}, 3$; moyen: $\mathrm{M}, 2,5$; moyen-bas: $\mathrm{M}^{-}, 2$; bas: $\mathrm{B}, 1$; infra-bas (= hors de la gamme habituelle du locuteur): IB, 0,5.

7 Conversation entendue dans le train, 31 mars 2003.
} 


\subsection{La (re)production orale}

Accomplie par le linguiste par exemple dans une communication à un colloque, la reproduction orale tient à la capacité de mimétisme, et qui plus est de mimétisme différé. On est en droit de se demander quel degré de précision les destinataires peuvent accorder à la justesse de la voix du producteur.

On retrouve là une circonstance qui se rencontre avec les étrangers, comme le signalait déjà par exemple Rousselot (1911: 83) : "L'homme instruit peut user d'une prononciation qui n'est pas de sa condition [...]. L'étranger, lui, ne possédant pas l'art [...] ne réussit qu'à nous faire rire". De même avec les acteurs lorsqu'ils font des erreurs de diction. Avec un texte imposé, qu'ils ont donc répété à l'avance, ils sont censés donner l'impression de parler de leur propre chef et de façon libre, avec une intention personnelle. Mais ils n'y réussissent pas toujours. C'est ce qui arrive en (3):

F- $\quad[\ldots]$ et il a emporté "tous les double-'jeu $/ 8$

S- $\quad$ ((à la cantonnade et en se frappant le front)) il est toc 'toc /

$$
\begin{array}{ll}
= & \text { "à mon avis/moi je vous dis qu' il est zinzin/timbré" } \\
\text { OU } & \} \\
=\text { "est-ce que vous pensez qu'il est devenu fou/qu'il a } & \} \\
\text { perdu la tête ?" } & \text { ? }
\end{array}
$$

En tant que téléspectateur, je n'ai pas été en mesure de comprendre ce que veut dire la petite fille (S) avec son "il est toc toc", car l'élévation de son fondamental ${ }^{9}$ sur la dernière syllabe est trop importante pour une constatation mais insuffisante pour une question.

Dans de telles situations, celui qui parle a bien sûr dans l'esprit une intention. Pour lui, elle est claire; mais sa transmission ne l'est pas. En (3), lorsqu'elle s'adresse à sa mère - et bien sûr aux téléspectateurs -, S veut-elle dire ce qu'elle pense de F, ou se pose-t-elle la question de savoir si F a toute sa tête?

\subsection{La cohérence textuelle}

D'autres difficultés encore sont inhérentes à la bonne restitution de ces corpus particuliers, et ce pour au moins 3 raisons:

a) l'absence du contexte d'énonciation, lorsque l'on entend les paroles mais qu'à la différence des interlocuteurs, on ne voit ni l'espace environnant de tout ce qui s'y trouve (personnes, objets, etc.) ni ce qui est dans le champ de vision de chacun d'eux;

b) l'absence de cotexte développé. Quand l'écoute, normalement fixée sur le contenu, est soudain détournée vers la forme en raison d'une variation significative, le linguiste se trouve devant un dilemme: retenir un maximum d'informations sur le point qui a attiré son attention (les attributs déterminants), ou essayer de se rappeler également les propos qui l'encadrent (les passages précédents et suivants). Car faire les deux à la fois relève de la mission impossible.

c) le rapport entre un écart marquant pour l'auditeur-linguiste et les habitudes énonciatives de l'émetteur, ainsi que la distance entre sa compréhension et l'effet produit sur le(s) 'vrai(s)' destinataire(s). C'est le cas par exemple pour certaines ambiguïtés et/ou polyvalences détectées par le spécialiste - même s'il a capté la suite immédiate du discours -, alors qu'elles sont résolues par le protagoniste effectif.

\footnotetext{
${ }^{8}$ Publicité pour des mousses au chocolat "double-jeu", sur M6, 25 janvier 2002 (donc avec des acteurs qui jouent un rôle, et non en situation d'énonciation 'réelle', spontanée). Le jeune garçon (ici F-) finit de raconter à sa mère - en présence de sa petite sœur - une histoire complètement abracadabrante d'extra-terrestre responsable de la disparition des petits pots de mousse au chocolat dans le frigo. La publicité se termine avec la réplique de la petite sœur (ici $\mathrm{S}$-).

9 Désormais Fo.
} 
Voyons par exemple ce qui se passe en (4):

(4) JPP- [...] c'est pas en'core à la déci'sion /

$\mathrm{Y}$ - $\quad$ Tu en as parlé avec 'X ?

JPP- $\quad$ 'oui / j(e l)u(i) ai d(e)mandé ce qu'i(l) pensait du do'ssier et il a pas mal réa'gi [...] ${ }^{10}$

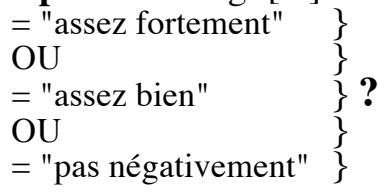

Je me suis jointe au groupe au moment où se tenait cette partie du dialogue. De plus, non seulement je ne savais pas qui était $X$ et sa façon la plus probable de réagir, mais encore "pas mal" a été articulé avec une sous-spécification sonore; c'est pourquoi je me suis trouvée dans l'incapacité de comprendre si cela signifiait:

- "assez fortement", c'est-à-dire que JPP avait noté chez X une réaction négative vive,

- ou "assez bien", ce qui aurait voulu dire que $X$ avait eu une réaction plutôt positive,

- ou encore "pas négativement", au cas où X s'était contenté de ne pas réagir de façon négative, en quelque sorte de façon neutre, ce qui aurait signifié que "pas mal" était la version familière de "ne ... pas mal".

En revanche l'allocutaire Y n'a manifesté aucune incompréhension et a donné des signes prouvant qu'il avait bien compris; ce qui amène à la conclusion qu'il fallait qu'il connaisse le dossier en question et le tempérament de la personne dont JPP parlait.

\section{Comment procéder?}

Comme on l'a vu avec les exemples (1) à (4), pour exploiter les corpus-surprise avec quelque sécurité des précautions sont indispensables, mais surtout l'application de plusieurs règles procédurales, qui sont celles de l'analyse perceptuelle des corpus enregistrés. J'exposerai les 2 principales.

\subsection{Premier principe}

Lorsqu'un passage contient un sens particulier en raison de la façon dont il a été prononcé, il convient de rédiger cette signification en détaillant au maximum tous les plans d'information qu'elle contient: appréciatif, méta-discursif, des actes illocutoires, de relation interpersonnelle, de place interactionnelle, de structuration conversationnelle, du travail de formulation. Pour obtenir la plus grande précision possible il est préférable de 'faire comme si', c'est-à-dire de tenter de se mettre à la place de quelqu'un qui devrait exprimer en mots toutes les facettes de ce qu'il veut transmettre à son interlocuteur - un peu comme il serait obligé de le faire si ce dernier était atteint de cette sorte d'aphasie (lésion du cerveau droit) "qui met dans l'incapacité de traiter les sous-entendus"11. On peut par exemple choisir un autre niveau de langue (un des niveaux plus soutenus ou au contraire "inférieurs", en fonction de la richesse lexicale existant pour le sens considéré).

Dans tous les cas éviter à tout prix la nominalisation, qui est toujours un piège fatal, comme le met en lumière le titre de l'exemple (5):

10 Un collègue, en réunion de Département, Chambéry 12 mai 1998.

11 Kaïl (2000). 
(5) Ordre

phrase 1: Ne quittez pas!

phrase 2: Ne coupez pas!

phrase 3: Ne fumez pas tant!

phrase 4: Ne me laissez pas seule!

phrase 5: Ne parlez pas tant!

phrase 6: Ne regardez pas cette "idiotie"!

phrase 7: Ne me demandez pas ça! ${ }^{12}$

Pour le locuteur natif de français lisant ces phrases, l'exercice ne devrait pas être intitulé "ordre": en effet, bien que tous ses items présentent un des types d'injonction existant effectivement - avec son verbe conjugué à l'impératif et, ce qui est obligatoire dans le mode de transmission écrit, un point d'exclamation final -, il ne contient aucun ordre véritable et regroupe en réalité 4 actes de langage au moins:

- les phrases 1 et 2 contiennent une requête et pourraient être accompagnées de la formule de politesse: "s'il vous plaît" ou des cris dont un équivalent serait: "alerte!" (appel d'urgence au SAMU par exemple);

- la phrase 3 traduit un reproche de même que 6, parmi les sens qu'on peut lui donner et 5, qui peut aussi être une supplication;

- la phrase 7 est elle aussi une sorte de supplication;

- enfin le sens de la phrase 4 est pour moi indécidable, car trop ambigu: on pense en effet à plusieurs signifiés différents selon les contextes qu'on leur imagine.

Il va de soi que dans le cas d'un corpus enregistré on ferait une contre-expertise pour valider la description élaborée: faire écouter le fragment à une ou mieux à plusieurs autres personnes de préférence non-linguistes, leur demander comment elles le comprennent et comparer les réponses pour voir si elles concordent. S'il y a des divergences, réexaminer chaque partie pour cerner au plus près de quel(s) aspect(s) du signifié il s'agit et en quoi il(s) diverge(nt), puis réécouter et de faire réécouter le passage dans le but de trouver une reformulation commune et homogène.

\subsection{Second principe}

Deuxième temps: pour décrire la réalité sonore qui est le signifiant d'un sous-entendu l'apprentissage d'une écoute différente est indispensable. L'objectif est d'arriver à faire abstraction de ses habitudes d'auditeur-locuteur natif, qui portent naturellement et logiquement à s'attacher aux informations données par celui qui parle. L'éducation nécessaire, qui conduit à savoir percevoir avec une 'oreille linguistique', ne se fait pas seul, mais avec un maître reconnu en la matière.

En tout état de cause on retiendra que les éléments principaux du protocole à suivre lorsqu'on possède des corpus enregistrés sont les suivants:

- l'analyse se fait par fragments n'excédant pas 2 secondes. Pour discerner au sein du flux syntagmatique des phénomènes parfois extrêmement ténus, la mémoire-tampon n'est pas efficace au-delà;

- chaque paramètre acoustique est étudié séparément des autres. Il est nécessaire d'isoler chacun d'entre eux des autres paramètres car la complexité de l'ensemble oral rend hors de portée une notation qui se ferait de façon globale. On se concentrera par exemple sur l'intensité $(\mathrm{dB})$ pour évaluer au plus juste le différentiel existant d'une syllabe à la suivante, ou d'un segment (consonne, voyelle ou glide) au segment contigu;

\footnotetext{
12 Exercice 6 d'une série dite "de prononciation", dans la méthode de français comme langue étrangère Archipel de Courtillon/Raillard (1983).
} 
- au minimum une nouvelle écoute sera faite plusieurs jours plus tard, pour compléments et vérifications, en espérant qu'on ne défera pas alors tout ce qu'on croyait avoir construit la fois précédente...

Avec les enregistrements, on ne peut prévoir à l'avance le nombre d'écoutes qui sera nécessaire pour arriver au meilleur résultat, mais ce dont on est sûr par avance c'est qu'il pourra être très élevé; on sait aussi avec certitude que les passages examinés seront imprimés à jamais dans la mémoire du chercheur!

\subsection{Deux prérequis essentiels}

a) La couche vocale possède une certaine autonomie par rapport aux supports lexicaux, en ce sens que les traits acoustiques ne s'appliquent pas automatiquement sur la totalité d'un mot, d'un groupe syntagmatique ou d'un groupe prosodique, et que de plus, dans un signifiant vocal, les indices n'ont souvent pas la même extension entre eux.

Là encore l'apprenti pourrait s'inquiéter, croire qu'il se trompe et invalider ce qu'il a entendu, au lieu de penser qu'il est peut-être tombé juste en identifiant une telle caractéristique.

b) Enfin un point très important à garder à l'esprit pour toute recherche de ce type, peut-être le plus important, est que le sens présent au travers des marques de la couche vocale du discours (le sens pragmatique) n'est pas lié à tel ou tel de ses traits, mais bien à leur combinaison, qui organise les indices en un signifiant stable pour le signifié considéré. C'est un défaut courant du débutant d'associer marque acoustique et signification, même s'il arrive parfois (rarement) qu'un sens pragmatique ne soit porté que par un seul indice sonore.

Pour illustrer ces 2 dimensions du problème, j'ai sélectionné 4 des nombreux messages que peut porter l'énoncé (6a) lorsqu'il apparaît dans des situations réelles. Nous constaterons combien les signifiants vocaux sont de natures diverses, ce qui nous permettra de prendre en partie la mesure de la complexité de leurs agencements.

(6a) elle est bien en bleu

Transcrite en orthographe traditionnelle, cette phrase s'avère avoir plusieurs sens potentiels, un seul apparaissant lors de telle énonciation selon les choix morphologique et syntaxique, en même temps qu'en fonction de l'intention, donc de l'acte perlocutoire qui y est attaché.

Chacun des sens possibles est exclusif des autres grâce à la présence des indices vocaux. En effet, le message final sera ici lié:

- aux emplois grammaticaux de "bien": comme adjectif, on peut le remplacer par "joli"; comme adverbe il a deux acceptions: "effectivement", et "trop";

- aux différents sens de "bleu": comme adjectif, il dénomme une couleur; comme substantif, c'est l'abréviation de la lexie complexe "bleu de travail";

- aux traits acoustiques concomittants:

- la liaison avec "bien". Elle est obligatoire quand le mot est adverbe et précède le mot auquel il se rapporte et se manifeste par la prononciation du segment [n] ("bien" =

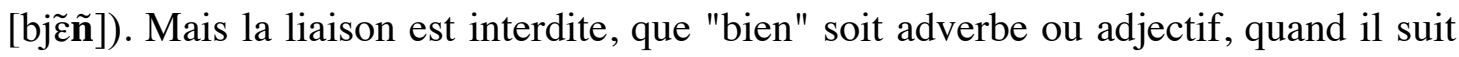
le mot auquel il se rapporte. On a alors "bien" = [bjẽ $]$;

- la présence d'une pause interne, ici entre "bien" et "en":

$>$ possible quand "bien" qualifie ce qui précède - ici "elle" -,

$>$ mais impossible quand "bien" a un effet sur sa droite, car le signe de ce lien grammatical est la liaison obligatoire entre "bien" et "en" - d'autant plus que c'est le seul indice dans cet énoncé, et donc qu'il est indispensable puisque discriminant -; 
- la durée de la pause interne (lorsqu'il y a pause): par rapport au locuteur, normale, longue, très longue ou courte à très courte;

- la hauteur tonale relative des syllabes. Elle est soit simple soit complexe, par la combinaison de tons simples (voir supra note 4);

- l'amplitude de l'intensité (dB), par diminution ou par augmentation;

- le(s) type(s) de groupe(s) prosodique(s) et son/leur(s) schéma(s) intonatif(s): progrédient (dit aussi continuatif) ${ }^{13} \sim$ terminal $^{14} \sim$ incident $^{15} \sim$ énumératif ${ }^{16} \sim$ non modulé $^{17}$.

D'une expression à l'autre, certains de ces traits présentent des similitudes qu'une traduction trop grossière ne rendrait pas visibles, ce qui ne permettrait plus de différencier les différentes versions.

Pour: "elle est bien en bleu", si l'on met à part les énonciations équivalant à: "elle est jolie en bleu", "elle est effectivement en bleu" , ou "je trouve qu'elle met vraiment trop de bleu!" , certaines combinaisons sonores nous montrent que des variations apparemment de faible ampleur peuvent entraîner des changements de sens importants.

Voyons la différence entre (6b), (6c) - qui est l'exemple effectivement entendu -, (6d) et (6e):

$$
\begin{gathered}
\text { elle est 'bien / en 'bleu ' } \\
\mathrm{M}^{+} \mid \mathrm{B}
\end{gathered}
$$

(6c) F- $[\ldots]$ moi j(e) te dirai mon a'vis a'prè:s mais 'toi sa co'pine tu la trouves "co'mment / physi'qu(e)ment j(e) veux 'di:re /

H- elle est 'bien' en 'bleu /18

$$
\mid \quad \mathrm{B} \underset{<\mathrm{E}>}{\mathrm{B}} \mathrm{M}^{-}
$$

F- ((stupéfaction puis rire) $)$

(6d) elle est 'bien' en "ble $\mathrm{u} /$

$$
\text { B } \quad \mathrm{B} \quad \mathrm{H}^{+} \text {à } \mathrm{M}
$$

(6e) elle est 'bien' ${ }^{\prime}$ en 'bleu

$\mathrm{M}^{-} \mid \mathrm{IB}$

En (6b), le premier groupe prosodique est continuatif, mais:

- "bien" a son lien grammatical à sa gauche, lequel interdit la liaison de "bien" avec la suite, ce qui à son tour permet la pause qui suit;

- le deuxième groupe est bas non modulé, alors qu'on l'attendait terminal.

Cette configuration fait que se dégage, au lieu d'un compliment clair, le sens pragmatique qu'on pourrait reformuler: "Je la trouve jolie, quand elle est en bleu (= c'est en bleu qu'elle est mise en valeur)". Pour autant, l'opinion de l'émetteur n'est pas négative.

\footnotetext{
13 Désormais GPC: sur la syllabe finale, Fo plus haut que la pénultième, ton plat.

14 Désormais GPT: sur la syllabe finale, Fo plus bas que la pénultième (1 quarte), ton plat.

15 Désormais GPI: Fo plus bas que le niveau moyen précédent (sauf la dernière syllabe), et remontée intonative (faible) sur la dernière syllabe; ce schéma intonatif est accompagné de: pause (ou pas de pause) initiale plus en général, pause finale.

16 Désormais GPE: sur la syllabe finale, ton complexe montant.

17 Désormais GPNM: intonation très légèrement descendante tout au long du groupe.

18 Conversation entre amis, novembre 2004.
} 
En (6c), le premier groupe a un schéma intonatif terminal; de plus, la pause interne est longue. Ces deux phénomènes combinés - qui interdisent la liaison interne - indiquent que "bien" s'applique à ce qui est à sa gauche. Ils induisent $\mathrm{F}$ à croire que "elle est bien" constitue un énoncé achevé, complet. Aussi, lorsque $\mathrm{H}$ dit "en bleu", F est-il obligé, après coup, de réinterpréter la première proposition. Il y a donc traitrise: le soudain revirement de $\mathrm{H}$ oblige $\mathrm{F}$ à reconsidérer ce qui précède comme le début d'un seul ensemble, dont le sens est tout différent de ce qu'il pensait d'abord. Le résultat est très désobligeant pour la personne dont il est question, car développé explicitement, on en déduit quelque chose comme: "Elle est regardable. Mais entendons-nous bien (= je précise): seulement quand elle est en bleu."

En (6d), si les hauteurs tonales jusqu'à "en" sont identiques à celles de (6c), déclenchant la même nécessité d'une relecture générale, celles de "bleu" ne le sont pas. L'ensemble contient une limitation supplémentaire, un vif retrait de l'énonciateur, l'équivalent de: "Elle est regardable. Mais attention! (= ne me faites pas dire ce que je n'ai pas dit!) seulement en bleu!"

En (6e), le premier groupe ressemble à celui de (6d), mais il n'est pas terminal; il est cependant suivi d'une pause longue (donc pas de liaison interne). Le deuxième groupe, très bas, est nommé dans le modèle de Morel/Danon-Boileau (1998: 28-32) un postrhème ${ }^{19}$. L'effet est une antiphrase, qu'on pourrait gloser: "Alors là, bravo, (ironiquement) quelle réussite! (= ça ne peut pas être pire!) En bleu, elle est plus que laide..."

Ces exemples expliquent l'importance qu'il y a à ne pas négliger le moindre écart: si les traits sont insuffisamment analysés ou avec une trop grande imprécision, cela peut amener à des contresens considérables. On le constate à nouveau avec l'exemple (7):

(7) [...] il y avait un $\mathrm{h}$ que j'avais pas prononcé à la française. ${ }^{20}$

Seule l'analyse précise des groupes prosodiques - et l'absence de pause avant "à la ..." permettent de décoder que JD veut dire qu'il n'a PAS prononcé le h, "car c'est l'usage en français de ne pas le faire", en réalisant trois groupes rythmiques, marqués par trois syllabes toniques, soit (7'),

[...] (il) y avait un 'h que j'avais "pas pronon'cé à la fran'çaise /

et NON (7"),

(7") [...] (il) y avait un 'h que j'avais "pas prononcé à la fran'çaise /

qui n'en a que deux et se reformulerait: "Je n'ai pas fait comme en français, où le h en question ne se prononce pas; je l'ai prononcé, comme on le fait en arabe", sens erroné pour cette occurrence particulière de ce locuteur particulier à ce moment-là.

\section{$4 \quad$ Remarques finales}

$\mathrm{Si}$, pour les partenaires d'un dialogue, une analyse conforme de l'implicite de l'énonciation de l'interlocuteur est importante en elle-même mais aussi parce que de sa réussite dépend la facilité avec laquelle se déroule la suite de l'échange, pour le linguiste, elle est déterminante autant quant à la compréhension de l'évolution des rapports entre les protagonistes, au travers de leurs réactions verbales et non-verbales, que par rapport à la vérification du bien-fondé de ses hypothèses et au développement de ses théories.

\footnotetext{
${ }^{19}$ C'est pour ces auteurs un cas particulier de l'incise; il arrive toujours en position finale d'un paragraphe ternaire. Il "vient à l'appui d'une position singularisée, sur laquelle l'énonciateur anticipe une rupture, donc toujours en situation de discordance énonciative [...]; souligne une position fortement égocentrée [...]; traduit le plus souvent une attitude polémique de l'énonciateur, liée à l'intrusion d'une donnée inattendue dans la situation de l'échange, souvent déclenchée par le récepteur".

${ }^{20}$ Un collègue, en réunion de DEA, 4 octobre 2000.
} 
Mais, sans parler du temps nécessaire à l'exécution de la mise en forme écrite des corpus-surprise, on a vu que l'apprentissage préalable du chercheur, comme ensuite la minutie du travail à accomplir et des précautions à prendre pour offrir des garanties de véracité à ces sortes d'exemples sont des contraintes fortes, et en même temps indispensables car dans ce domaine, ce qui semble une évidence est une des plus grandes causes d'erreur. Autrement dit, transparence supposée rime ici pratiquement toujours avec illusion.

Il est donc capital d'avoir acquis les savoir-faire pour se lancer dans cette aventure que représente l'analyse perceptuelle de la prosodie. La fiabilité des données n'est qu'à ce prix. Toute la question est dès lors de savoir si l'on considère que le résultat attendu justifie la quantité d'énergie à dépenser pour y parvenir.

\section{Conventions d'écriture}

(1) $=$ numéro d'ordre des exemples

$\mathrm{X}-\quad=\quad$ émetteur en position d'énonciateur premier

(Y) = émetteur en position d'énonciateur second

[...] = le tour de parole a commencé avant le fragment/se poursuit après le fragment

( ) = son/syllabe non prononcé(e)

$(())=$ réactions non-langagières

P $=$ consonne occlusive glottale

$:: \quad \quad=\quad$ allongement du son: normal/plus long

1 $=$ accent tonique

" = accent "d'insistance"

/ $=$ pause

$\$ \$$

$=\quad$ zone de chevauchement de paroles

$\$ \$$

$<\mathrm{W}>\quad=\quad$ signe vocal

\section{Bibliographie}

Adolphs, Svenja (2008): Corpus and Context. Investigating pragmatic functions in spoken discourse. Philadelphia. (= Studies in Corpus Linguistics 30).

Bilger, Mireille (ed.) (2000): Corpus. Méthodologie et applications linguistiques. Paris. (= Bibliothèque de L'INaLF. Les français parlés - textes et études 3).

Blanche-Benveniste, Claire/Jeanjean, Colette (1987): Le français parlé, transcription et édition. Paris.

Bybee, Joan (2001): Phonology and language use. Cambridge. (= Cambridge Studies in Linguistics 94).

Bregje, Christina de Kok (2008): "The role of context in conversation analysis. Reviving an interest in ethno-methods". Journal of pragmatics 40/5: 886-903.

Cappeau, Paul/Gadet, Françoise (2007): "Où en sont les corpus sur les français parlés?" Revue française de linguistique appliquée XII: 129-133. 
Courtillon, Janine/Raillard, Sabine (1983): Archipel 2. Unites 8 a 12. Francais langue etrangere. Cours crédif. Paris.

Delais-Roussarie, Elisabeth/Yoo, Hiyon (2009): "Transcrire la prosodie. Un préalable à l'échange et l'analyse de données". Présentation lors de la journée Conscila Regards croisés sur la prosodie du français Paris 2009.

Kaîl, Michèle (2000): "Enjeux actuels de la psycholinguistique développementale". Présentation lors du IIIe colloque international de la COFDELA : Perspectives cognitives en linguistique et didactique des langues Avignon 2000.

Kerbrat, Catherine (2008): "La place de l'interprétation en analyse du discours en interaction". Revue de sémantique et pragmatique 19/20: 31-49.

Laks, Bernard (2008): "Pour une phonologie de corpus". Journal of French Language Studies 18: 3-32.

Martin, Philippe/Avanzi, Mathieu (2007): "WinPitch. Un logiciel pour la détection automatique des proéminences accentuelles dans les corpus oraux". Présentation lors du colloque CILPR Innsbruck 2007.

McHoul, Alec (2008): "Questions of context in studies of talk and interaction. Ethnomethodology anc conversations analysis". Journal of pragmatics 40/5: 823-826.

Morel, Mary-Annick/Danon-Boileau, Laurent (1998): Grammaire de l'intonation. L'exemple du français. Gap etc. (= Bibliothèque de faits de langues. Linguistique).

Post, Brechtje/Delais-Roussarie, Elisabeth/Simon, Anne Catherine (2006): "IVTS, un système de transcription pour la variation prosodique". Bulletin de la Phonologie du Français Contemporain 6: 51-69.

Rittaud-Hutinet, Chantal (1995): La phonopragmatique. Bern. (= Sciences pour la communication 45).

Rittaud-Hutinet, Chantal (2007): "Vous avez dit: 'c'est bien ça'?, ou: variations vocales et sens pragmatique". In: Pifarré, Alexandra-Flora/Rutigliano-Daspet, Sandrine (eds.) (2007): Le lien, la rupture. Chambéry: 57-67. (= Langages, littératures, sociétés 3).

Rittaud-Hutinet, Chantal (2007): L'homophonie. Limoges.

Rittaud-Hutinet, Chantal (2008): "Consensus, conflits et négociations implicites". Présentation lors du colloque international Les voix du français: usages et représentations Oxford 2008.

Rittaud-Hutinet, Chantal (2008): "Fonctions syntaxiques et pragmatiques de la prosodie". In: Collin, Catherine (ed.): Travaux linguistiques du CerLICO: grammaire et prosodie 1. Rennes: 63-79.

Rittaud-Hutinet, Chantal (2008): "Le langage non verbal, ou: Signes vocaux et sens du message". Revue de sémantique et pragmatique 19, 20: 71-88.

Rousselot, Pierre-Jean (1911-1912): "Dictionnaire de la prononciation française". Revue de phonétique 1: 79-92; 2: 159-191, 260-285. 